\title{
Dispositivos de la globalización: la construcción de grandes proyectos urbanos en Ciudad de México
}

\begin{abstract}
Alfonso Valenzuela. Universidad de California en Berkeley; Universidad Autónoma del
\end{abstract} Estado de Morelos, Morelos, México.

RESUMEN | El impacto territorial de la globalización se ha traducido en la construcción de grandes proyectos urbanos que han servido como puntos de contacto al interior de las ciudades ligadas a los circuitos de capital. Mediante una aproximación gramsciana, analizamos el fenómeno de los megaproyectos en América Latina tomando como caso de estudio Santa Fe, en el poniente de Ciudad de México, como un parque corporativo inscrito dentro de los flujos hegemónicos globales. Desarrollamos para el análisis el concepto de dispositivo de la globalización, como el espacio urbano articulador del capital global. Dichos dispositivos funcionan a una velocidad distinta al resto de la ciudad circundante y se revelan como un instrumento del poder económico vertical en medio de una horizontalidad distante, empobrecida y ajena a los flujos globales.

PALABRAS CLAVE | globalización, proyecto urbano, reestructuración territorial.

ABSTRACT | The territorial impact of globalization materializes with the construction of large urban projects, which serve as contact points within cities related to the circuits of capital. By means of a gramscian approach we use Santa Fe - a megaproject in the western part of Mexico City - as a case study of a corporative development project inscribed in global hegemonic flows. Building upon the concept of global devices to designate such projects, they become the urban space link to global capital. However, such devices operate at a different speed than the rest of the city, as instrument of a vertical economic power in the midst of a distant, impoverished and unrelated to global flows horizontality.

KEY WORDS | globalization, urban project, territorial restructuring

Recibido el 13 de junio de 2011, aprobado el 23 de febrero de 2012

Email:aval@uaem.mx 


\section{Introducción: Globalización y flujos diferenciales}

En "Los espacios de la globalización" (1993), Milton Santos establece una distinción que nos parece fundamental para precisar el impacto territorial de la globalización. Básicamente, desmitifica la difundida noción de que la globalización comporta una homogeneización del espacio, cuando en realidad vuelve el territorio más heterogéneo, diversificado e incluso dividido (p. 73). En segundo término - y quizá su aportación más importante-, identifica dentro de la globalización la creación de horizontalidades y verticalidades; las primeras ligadas al ámbito local y cotidiano de los habitantes, mientras las segundas corresponden a los puntos de articulación de los actores hegemónicos, es decir, a los llamados "vectores de integración jerárquica regulada" (p. 74). Entre las horizontalidades y verticalidades existe una tensión que supone una constante adaptación de las formas y las normas. Sin embargo, aun cuando en ocasiones las verticalidades se adaptan a las particularidades de algunos lugares, los actores hegemónicos (firmas o corporaciones multinacionales) tienen la facultad de trasladar sus vectores de acuerdo con las necesidades del mercado, pudiendo generar crisis locales sin recibir los costos políticos correspondientes.

Santos se ocupó del impacto territorial producido por la globalización desde principios de la década de los noventa, designando el nuevo fenómeno como la "informacionalización del espacio" (Ibíd., p. 71). Además, advertía el hecho de que dichos lugares no solo responden a los intereses de los actores hegemónicos de la economía y de la sociedad, sino que incluso les permiten incorporarse con toda autoridad a las corrientes de la globalización. Si bien otros autores han identificado un espacio de flujos globales con acento en las dinámicas simultáneas de concentración y dispersión (Castells, 1989; Sassen, 1991; Hall, 1998), Santos destaca el hecho de que existen distintos niveles, intensidades y orientaciones de dichos flujos. En otras palabras, distingue los flujos hegemónicos de los flujos hegemonizados, señalando que los primeros son rápidos y eficaces, mientras los segundos son más lentos y dependientes. Por tanto, siempre Santos, la articulación del espacio global se produce mediante redes desiguales yuxtapuestas a distintas escalas y niveles, un patrón en el cual únicamente los actores hegemónicos utilizan todas las redes y dominan todos los territorios (p. 73). Esta aproximación gramsciana se demuestra particularmente útil para analizar el fenómeno de los megaproyectos en América Latina en general, y Santa Fe - al poniente de Ciudad de México - en particular. Santa Fe se puede definir como un parque corporativo inscrito dentro de los flujos hegemónicos globales y con una velocidad distinta al resto de la ciudad circundante. Con el paso de los años, Santa Fe se consolidó como un polo no solo de servicios, sino también inmobiliario, así como un espacio de poder económico en medio de la horizontalidad distante, empobrecida y ajena al flujo global.

Los grandes proyectos urbanos tienen diversos impactos importantes en el territorio (Lungo, 2004, p. 17). Por una parte, se altera el valor del suelo mediante el cambio de usos que generalmente acompaña a un proyecto; se modifican la morfología y las funciones de extensas áreas de la ciudad; y finalmente, se establece un modelo de gestión territorial distinto al tradicional. Estos proyectos son particularmente 
atractivos para el capital, por su capacidad de generar una valoración exponencial del suelo que eventualmente formará parte de un mercado inmobiliario internacional ${ }^{1}$.

El concepto de proyecto urbano surgió en Europa en la década de los setenta, como una intervención pública sobre un sector de la ciudad, pero que forma parte de una visión global mediante la cual se intenta dar respuesta a los requerimientos sociales y económicos de sus habitantes. En México, y en la mayor parte de los países en América Latina, los grandes proyectos urbanos se convirtieron en instrumentos clave de planificación urbana justo después de la segunda generación de reformas estructurales impuestas por el Fondo Monetario Internacional a finales de la década de los ochenta (Stahl, 1994, p. 56). Este tipo de proyectos atrajo el interés de inversionistas y desarrolladores inmobiliarios toda vez que las grandes operaciones urbanísticas garantizaban la rápida valoración del suelo, obras de infraestructura mayores y la progresiva privatización de la gestión urbana. En esta línea, y de acuerdo con Harvey (1989, p. 7), a finales de la década de los ochenta la administración urbana pasó de tener un enfoque gerencial a otro empresarial, asumiendo los valores corporativos de competitividad, mercadeo y eficacia. Sin embargo, los criterios referidos empezaron a traducirse en un desarrollo territorial inequitativo, en la priorización de áreas particulares y en asociaciones público-privadas con una base esencialmente especulativa.

Es importante destacar que si bien los grandes proyectos urbanos han evolucionado en las últimas dos décadas para incorporar criterios de equidad, integración, articulación y mejoramiento de las condiciones de vida de la población mediante la creación de espacios públicos, equipamientos culturales y nuevas centralidades, Santa Fe estuvo orientada en su totalidad hacia el establecimiento de un enclave financiero-inmobiliario del más alto nivel socioeconómico. Tal dirección responde a la hegemonización de la visión neoliberal de la gestión urbana, que enfatiza - dentro de la dimensión política- la necesidad de construir ciudades competitivas en el ámbito de la globalización con el fin de captar inversiones externas, en detrimento de ciudades más equitativas, inclusivas y justas.

\section{Neoliberalismo y hegemonía en Ciudad de México}

De acuerdo con Gramsci (1984), los gobiernos democráticos actúan con cierto grado de consentimiento de la gente que gobiernan, lo cual implica un continuo reposicionamiento entre gobernante y gobernados (p. 17). A principios de los años ochenta, México sufrió distintas crisis que llevaron a una reconfiguración de dicha relación, en detrimento de las conquistas sociales. Primero se produjo una crisis de tipo económico y financiero que derivó en la devaluación del peso en 500\% para finales de dicha década (García Fonseca, 2003, p. 17); después siguieron los esquemas de "ajuste estructural" dictados por el Fondo Monetario Internacional; y, finalmente, un terremoto devastó la ciudad central de la capital a mediados de di-

1 Por ejemplo, algunos de los desarrolladores, como GICSA, tienen proyectos no solo en Santa Fe, sino similares en Naya Miami Beach, Aloft Aventura y Everglades en Florida. 
cha década. Si bien las políticas neoliberales y privatizadoras se materializaron con el presidente Carlos Salinas de Gortari (1988-1994), la administración anterior sentó las bases para un giro desde un centro populista y social hacia una derecha empresarial (Luna, Millán \& Tirado, 1985, p. 217).

Durante el sexenio de Salinas de Gortari se agudizó la disparidad socioeconómica. En palabras de Salinas y Tavera (2007), "las desigualdades intersectoriales e intrasectoriales configuraron la concentración del ingreso y el producto en una elite de empresas y familias" (p. 277). De manera concurrente, se firmó el Tratado de Libre Comercio de América del Norte (TLCAN) y comenzó un proceso acelerado de desregularización de los mercados financieros. El consentimiento de la población estuvo ligado en un principio al ideal de competitividad global, internacionalización y, finalmente, la anhelada entrada al primer mundo. Sin embargo, el reposicionamiento entre gobernantes y gobernados se vio alterado por algunos grupos contrahegemónicos como los zapatistas, que cuestionaron desde un inicio el tipo de desarrollo abanderado por los grupos en el poder (Hoetmer, 2009, p. 85).

Es importante destacar que al inicio de la administración salinista se echaron a andar varios de los llamados megaproyectos: Santa Fe, la comercialización de la zona residencial de Polanco, el rescate de zonas lacustres en Xochimilco, el programa integral de Valle de Bravo y el proyecto Alameda, entre otros. Quizá entre dichos megaproyectos el que tendría mayores similitudes con Santa Fe es el proyecto Alameda, donde se impulsaban desarrollos inmobiliarios de alto nivel destinados al sector financiero y corporativo global. Surgido como respuesta a la destrucción de 34.000 edificios en la zona con el terremoto de 1985 (Perló Cohen, 1999, p. 283), con él se pretendía iniciar la recuperación del perímetro histórico de la capital. La iniciativa tuvo su origen en las altas esferas del gobierno y para el caso se convocó a reconocidos urbanistas, entre los que destacaban Luis Ortiz Monasterio, Eduardo Terrazas, Juan José Díaz Infante y Mario Pani, quienes recomendaron de entrada la inmediata demolición de las trece manzanas y su posterior reconstrucción, a imagen y semejanza de los programas de renovación urbana en las ciudades de Nueva York, París y Londres (Villalpando Ricardo, 1993, p. 142). Solo las altas densidades habitacionales y comerciales que tenían algunas de las manzanas llevaron a descartar dicha alternativa.

Se optó, entonces, por crear un fideicomiso privado con el objeto de "coordinar e impulsar la promoción inmobiliaria de propietarios e inversionistas", el cual intentaría quintuplicar el área que se intervendría, con el objeto de cubrir los requerimientos de vivienda agudizados por el sismo, cuando en realidad existía un proyecto de desarrollo inmobiliario de alto nivel para la zona. La racionalidad del proyecto fue presentada por el entonces director del recién creado Fideicomiso Alameda: "De las trece manzanas que conforman el Proyecto Alameda, más de la mitad de los terrenos o edificios de la zona se encuentran baldíos, en proceso de demolición, abandonados o con construcciones efímeras, y en contraste, la infraestructura de la zona (de las mejores equipadas de la ciudad) se encuentra francamente subutilizada, lo que propicia un dramático aceleramiento en su deterioro y un 
creciente vacío en la actividad económica" (Villalpando Ricardo, 1993, p. 141). El proyecto fue asignado al Grupo Danhos - dirigido por Jorge Gamboa de Buen-y a Reichmann International, con la idea de crear un centro financiero "estilo Manhattan" (La Jornada, 2005). El proyecto tuvo que rehacerse para integrar las necesidades de los residentes, frente al rechazo generalizado tanto de los especialistas como de los habitantes de la zona. Sin embargo, justo antes del término de la administración salinista, se echaron abajo los acuerdos anteriores y eventualmente se construirían únicamente los edificios públicos más comprometidos con el Gobierno de la Ciudad, como la Secretaría de Relaciones Exteriores, el Tribunal Superior de Justicia y el Archivo General de Notarías, así como los proyectos inmobiliarios más redituables, como el hotel Sheraton Centro Histórico, y los residenciales Plaza Juárez y Puerta Alameda.

\section{Santa Fe: la construcción de un dispositivo de la globalización}

Santa Fe fue creada a partir de la necesidad de contar con lo que De Mattos (2001) llama los "artefactos" de la globalización y mediante los cuales se busca generar puntos de contacto con la economía mundial (p. 40). Sin embargo, dicha caracterización es limitante, en el sentido de que la noción de "artefacto" remite a objetos estáticos, máquinas o aparatos. Proponemos a cambio el concepto de "dispositivo" el cual integra una dimensión dinámica, en cuanto mecanismo para producir una acción prevista. Si bien los megaproyectos tienen, por una parte, la función de servir como nodos focales dentro de una red global, también es cierto que son instrumentales para llevar a efecto el proyecto neoliberal hegemónico (Harvey, 2007, p. 35). En el campo de la informática, los dispositivos se refieren a estructuras sólidas que se conectan entre sí para alcanzar un objetivo, siempre y cuando se cumpla con las reglas de configuración y diseño. En el caso de los megacomplejos corporativos como Santa Fe, el diseño es simple, en el sentido de que solo responden a las necesidades de un sector particular de la población, dejando fuera a los que no están directamente involucrados, y desvinculándose de la ciudad misma.

La noción de dispositivo tiene también otra acepción, según la cual se refiere a un "esquema para engañar, estratagema o artificio" (Merriam-Webster, 1993). Si bien es discutible que este tipo de proyectos sean concebidos desde un inicio como instrumentos para confundir, no podemos negar que para su promoción y operación se ha utilizado una vasta gama de estratagemas y artificios destinados a identificarlos como iniciativas de conservación ecológica, de conexión global o como palanca para el desarrollo nacional.

En el marco descrito, Santa Fe se constituye como enclave global bajo el modelo sugerido por Sassen (1991, p. 346) y ampliado por Castells y Borja (1997, p. 249), en el sentido de que las corporaciones multinacionales demandan no solo las infraestructuras necesarias para la competitividad global, sino que también requieren un entorno urbano atractivo que pueda acomodar los recursos humanos especializados que dichas corporaciones ocupan. Las elites tecnócratas y gerenciales esperan 
encontrar centros educativos y comerciales de alto standing, aeropuertos, comunicaciones y sitios para el deporte y la recreación, siempre en entornos cerrados. A fin de cuentas, los enclaves socioeconómicos exclusivos dentro de realidades tan dispares y fragmentadas como son las ciudades latinoamericanas, se constituyen de manera estructural como expresiones urbanas divisorias excluyentes y fortificadas.

El plan maestro de Santa Fe fue concebido en su dimensión política por el entonces presidente Carlos Salinas de Gortari y el regente Manuel Camacho Solís, como parte de una serie de megaproyectos de desarrollo, inspirados en el área de La Defénse en París y presuntamente ligados al magnate norteamericano George Soros. El desarrollo se interrumpió hacia 1994 a raíz de la crisis financiera que azotó al país y se reanudó a principios de 2000. El plan se apoyó ideológicamente en un supuesto rescate ecológico de la zona, que al final nunca se llegó a implementar. En el documento ejecutivo se planteaban como objetivos "preservar y mejorar las áreas naturales en barrancas, reforestar las zonas con deterioro ecológico, sanear las cuencas hidrológicas, garantizar la recarga de los mantos acuíferos y recuperar a mediano plazo las áreas destinadas al basurero y relleno sanitario" (López Zárate \& Ochoa Méndez, 1995, p. 60). Con el paso de los años el interés conservacionista se fue diluyendo, y a pesar de que se menciona la importancia de la calidad ambiental, la creación de espacios públicos apaisados y de los programas de reforestación, el único parque público (la Alameda Poniente) tuvo que ser clausurado, debido a las emanaciones tóxicas provenientes del basurero sobre el que se construyó sin haberse llevado a cabo los trabajos de saneamiento necesarios. Históricamente, el concepto de sustentabilidad ha sido utilizado de manera extensiva en México para justificar agendas políticas encaminadas a mantener la estructura socioeconómica vigente (Valenzuela, 2011, p. 291). En el caso de Santa Fe, en un inicio se argumentaba que la zona había sido sobreexplotada con la extracción de arena y grava como insumos básicos para la construcción, a partir de lo cual se establecía en el mismo plan el destino de una cuarta parte del predio - equivalente a 215 hectáreas - como zona de preservación ecológica y áreas verdes, con especial atención a las cuencas y barrancas. Con el paso de los años la explotación minera (sobre todo arena) siguió con el ritmo previo y se utilizó precisamente para la construcción de los desarrollos inmobiliarios.

En un origen, el gobierno del Distrito Federal adquirió los terrenos a un precio de suelo agrícola, para venderlos después a los desarrolladores a un precio alzado de suelo urbano, utilizando parte de las ganancias para construir las infraestructuras urbanas correspondientes, así como equipamientos dirigidos a los sectores medios altos de la ciudad: la remodelación del Auditorio Nacional y del Zoológico del parque de Chapultepec, la creación de un Museo del Niño (de paga), así como la operación de los servicios públicos de emergencia y localización telefónica (Locatel). Sin embargo, es notable que durante el desarrollo de Santa Fe nunca se haya cuestionado el hecho de que el gobierno de la ciudad no capturara los incrementos en el valor del suelo generados por una intervención de carácter eminentemente público, aun cuando el capital fuera mayormente privado. 
Santa Fe es un caso paradigmático en que un gobierno, actuando en el ámbito administrativo de la ciudad, elige la opción de uso del suelo que representa los mayores rendimientos de capital dentro de la más pura lógica del sector privado. El Plan Maestro de Santa Fe comprendía el diseño y ubicación de oficinas corporativas de varias de las mayores multinacionales globales, pero sin considerar el tejido social y espacial que sustenta un crecimiento urbano equilibrado. Así, el desarrollo de Santa Fe fue determinado por los "productos inmobiliarios" que pudiera localizar exitosamente en el mercado de capital (Valenzuela, 2006a). Uno de los productos inmobiliarios paradigmáticos de segunda generación es City Santa Fe, el cual se promocionó de manera extensiva con la leyenda "Bienvenidos a la civilización: exclusividad con la mejor localización" (Jones \& Moreno-Carranco, 2007, p. 152). Para dicho desarrollo se programó la construcción de 42.000 metros cuadrados distribuidos en diez torres con 1.500 departamentos cada una. Las torres se agruparían por distritos interconectados mediante un corredor exterior (City Park), al que se integrarían jardines y zonas de esparcimiento. El complejo califica como parte de los nodos globales autocontenidos donde cada torre cuenta con gimnasio, alberca, spa, salón de usos múltiples, guarderías, áreas verdes y de recreación. Los usuarios tienen acceso a un City Walk con tiendas de autoservicio, boutiques y restaurantes, "todo bajo un sistema inteligente de seguridad con la más avanzada tecnología" (Akele, 2007, p. 1), complementado con un hotel de Gran Turismo dentro del mismo conjunto. El complejo se sustentó en la participación de arquitectos de reconocimiento internacional como la firma Pinififarina, Bernardo Gómez Pimienta, Francisco Serrano y Michel Rojkind. Concluye el promocional del proyecto: "así es como City Santa Fe llegará dentro de muy poco para romper paradigmas, asombrar a la ciudad entera y brindar una oportunidad única de propiedad para quienes valoran la naturaleza, belleza, comodidad y seguridad".

Si bien la promoción del proyecto coloca el énfasis en el sentido cualitativo del entorno, de hecho está cimentado en el valor de mercado. A este respecto, lo que resultó en verdad notable fue cómo la crisis financiera que sufrió Estados Unidos en 2008, incidió en que City Santa Fe (y la desarrolladora GICSA) se vieran obligadas a detener la construcción del proyecto, lo que derivó en cientos de demandas por incumplimiento del contrato de compraventa, además de numerosas infracciones a las normatividades y reglamentos oficiales (Reforma, 2010). No obstante la crisis referida, los nuevos desarrollos siguen multiplicándose con fórmulas de venta parecidas, como recientemente lo muestra el Corporativo Península, que ofrece actualmente un edificio "de marca" al conjugar los talentos de Teodoro González de León, Jon Jerde, Enrique Norten y la firma SOM para la creación de edificios "icónicos" en la zona. Dicho corporativo se presenta como "un espacio en donde la exclusividad, comodidad y elegancia son invitados todos los días" (Península Santa Fe, 2011), y en el cual los edificios ofrecen instalaciones ahorradoras de energía y agua, para sugerir su compromiso con el ambiente. Las velocidades diacrónicas que refiere Santos (1993, p. 73) para los circuitos de capital siguen lógicas alternas que desembocan no tanto en un discurso de nacionalismos (Sassen, 2003, p. 2), sino más bien en el fortalecimiento de las clases hegemónicas globales. 


\section{La privatización de la gestión pública}

A partir de la experiencia de reconversión urbana de la ciudad de Barcelona, los grandes proyectos urbanos toman como referencia el carácter polivalente y estratégico de dicha operación, incorporando desde su origen una participación ciudadana efectiva que permita pasar del proyecto urbano al proyecto de ciudad (Castells $\&$ Borja, 1997, p. 255). En el caso de Santa Fe, la participación social tuvo un proceso sui generis, debido a que la Asociación de Colonos Zedec de Santa Fe, A.C. se formó con los primeros inversionistas que establecieron sus corporativos en el desarrollo, y si bien cuenta en la actualidad con más de cincuenta asociados activos (entre los que se encuentran empresas, restaurantes, instituciones educativas, desarrollos habitacionales y centros comerciales), ni siquiera se menciona a los habitantes de las zonas residenciales (Colonos Santa Fe, 2011).

Utilizando la figura de una empresa público-privada, se asignó la gestión del proyecto a Servicios Metropolitanos S.A. de C.V. (Servimet), utilizando las Zonas Especiales de Desarrollo Controlado (Zedec) como instrumento urbanístico para la planeación de los usos del suelo. La Zedec Santa Fe se creó en 1987 con el objeto de permitir "el mejoramiento y rescate de la zona, con una reglamentación estricta del uso del suelo, que aprovechara su localización, infraestructura, suelo y elementos de acondicionamiento" (López Zárate \& Ochoa Méndez, 1995, p. 57). El cambio de modelo de gestión donde una empresa se hace cargo de los servicios, el mantenimiento y la vigilancia, corresponde al proceso identificado por Lungo (2004, p. 18) como característico de los megaproyectos, que incide en la atomización de las responsabilidades públicas. En este caso, además, dicho modelo contribuyó a consolidar los procesos de fragmentación socioespacial respecto de las zonas populares aledañas, con las que ya desde el origen se planteó — de manera tácita- el tener el menor número de vínculos posibles. Servimet se creó inicialmente como una de las primeras empresas público-privadas que, utilizando capital privado, se regía mediante las leyes y disposiciones de obras públicas del entonces Departamento del Distrito Federal. Es importante señalar que, en el mejor de los casos, dichas asociaciones utilizan figuras como las empresas de capital mixto, los fideicomisos y las corporaciones para el desarrollo urbano en la recuperación de centros históricos, captación pública de plusvalías o en la generación de empleos y recursos locales. Sin embargo, por su misma naturaleza, el interés del capital privado se orienta hacia el desarrollo de espacios con elevado potencial comercial y alto retorno financiero, evitando responsabilidades políticas así como priorizando los aspectos físicos de la renovación urbana por encima de los económicos, sociales y culturales.

De acuerdo con el entonces coordinador de asesores del director general de Servimet, el Plan Maestro de Santa Fe contemplaba "un desarrollo de excelencia, que atiende a criterios ecológicos, viales y urbanísticos de primer nivel, con la premisa de no olvidar criterios comerciales que permitan al proyecto ser autofinanciable y producir utilidades que se reviertan a la ciudad de México" (Barros Nock, 1993, p. 127). Si bien la venta del suelo generó ingresos, lo cierto es que no se recuperó la 
plusvalía generada por la intervención pública y financiada con recursos fiscales del resto de la ciudadanía. En cambio, el Departamento del Distrito Federal, junto con Servimet, se encargaron de la construcción de laterales a la carretera federal 15 (de cuota), de un sistema de puentes y varios tipos de conexiones internas al desarrollo, permitiendo "el acceso expedito a bosques de Las Lomas y a toda esa zona de altos ingresos de la ciudad" (Barros Nock, 1993, p. 128).

De acuerdo con Jones y Moreno-Carranco (2007, p. 151), Servimet fracasó en su función como gestor de servicios e infraestructura, delegando la autoridad y responsabilidad a la Asociación de Colonos existente. Es así como a partir de ese momento, la asociación creó un fideicomiso que tendría como objetivo "establecer las bases de colaboración y condición bajo las cuales el Gobierno del Distrito Federal realizará las aportaciones de recursos al fideicomiso, proporcionará las facilidades administrativas para que tramite y obtenga permisos y licencias para llevar a cabo obras, comprometiendo a la Asociación de Colonos a dotar de servicios públicos y realizar obras de urbanización y mejora para el área urbana de Santa Fe, procurando que los colonos estén al corriente en el pago del impuesto predial" (Colonos Santa $\mathrm{Fe}, 2011)$. En esta declaración de principios no solo se trasluce la delegación de facto de las atribuciones propias de gobierno, como son las obras, los servicios y la seguridad, sino que se manifiesta en tono triunfante que "por primera vez en México se logró una autoadministración privada, dirigida por la Asociación de Colonos de Santa Fe, por lo que la zona no pertenece a ninguna delegación” (Ibíd.). Esta organización es, entonces, una expresión paradigmática de la privatización de la gestión pública en la cual se consolida un circuito alterno a la realidad circundante, en buena parte autónomo, lo cual permite y justifica un tratamiento distinto al resto de la ciudad.

Solo después de seis años del funcionamiento del fideicomiso, el Gobierno del Distrito Federal (GDF) puso en tela de juicio la asignación de una partida del presupuesto para 2011. Esta decisión respondía a un intento - si bien tardío- de recuperar uno de los sectores de la ciudad con mayor plusvalía, así como restablecer los poderes delegacionales que hasta entonces se mantenían al margen. En este sentido, mientras que en las colonias circundantes las infraestructuras carecen del mantenimiento básico, en una de las avenidas principales de la zona corporativa se inauguró una veintena de esculturas monumentales realizadas por renombrados artistas, generando fricciones y descontento entre los habitantes de las colonias populares adyacentes (La Jornada, 2010b).

Si bien el GDF ha cuestionado la insuficiente transparencia en el manejo de recursos en el modelo de gestión de Santa Fe, el conflicto de fondo tiene que ver con la gobernabilidad del territorio. La zona pertenece formalmente a las delegaciones de Álvaro Obregón y de Cuajimalpa, pero en la práctica opera de manera independiente de la administración pública de ambas, lo que la convierte en una zona de excepción, un verdadero nodo global que no está ni siquiera ligado a la estructura político-territorial de la ciudad. Si bien se especuló sobre la eventual desaparición 
del Fideicomiso Santa Fe por utilizar un esquema de "administración privada" donde se manejan recursos públicos, pero sin ser auditable por tratarse de un ente privado, finalmente se le asignó el equivalente a diez millones de dólares para el ejercicio de 2011 (La Jornada, 2010a). Durante la presentación ante el actual jefe de gobierno capitalino del informe anual de la Asociación de Colonos Santa Fe, destacaban que el modelo del fideicomiso "es altamente eficiente y que derivado de sus instrucciones está en vías de que se pueda institucionalizar para asegurar su permanencia y efectividad en el futuro". Dicho modelo tendrá implicaciones de largo alcance, porque equivale a legitimar la privatización de la gestión pública basándose en el argumento de que la Asociación mantiene una disciplina rigurosa entre sus miembros respecto del pago del impuesto predial, y que incluso se compromete a "hacer muy evidente la retribución en servicios urbanos que esta comunidad se merece" (Boletín Colonos Santa Fe, 2011, p. 7). Por tanto, el tema en cuestión no es tanto la eficiencia en la gestión, sino las limitantes de dicho modelo para atender una zona con altos contrastes socioeconómicos, donde además de los corporativos, centros educativos y empresas, se incluya a los habitantes tanto de las zonas residenciales como de los barrios populares. Hasta ahora, la problemática territorial ha sido ajena a la administración delegacional, para la cual la solución consiste en encontrar un nuevo marco jurídico, actualizar el Plan Parcial de Desarrollo de Santa Fe y duplicar así el volumen de construcción existente (Ibíd.).

La falta de representatividad de los residentes se hizo patente hace un par de años con el surgimiento de un movimiento ciudadano alternativo en torno a la defensa del único parque existente en la zona. Conformados como la asociación civil Nuestro Parque Santa Fe, los residentes han planteado la designación de dicho predio como parque público ya desde el Plan Maestro Santa Fe original, y se posicionan en contra de la construcción de otro tipo de parque propuesto, "Garden Santa Fe", que comprende un centro comercial con 65.000 metros cuadrados de construcción y estacionamiento subterráneo para 1.600 automóviles. Si bien el desarrollo es subterráneo, el parque se reduce a las áreas residuales entre los conos de luz que darán iluminación natural al centro comercial. El proyecto - al igual que el mismo Santa Fe en su momentose promueve como un edificio certificado como sustentable, "comprometido con el medio ambiente" (uso de celdas solares, tratamiento de agua y otros sistemas de bajo costo que no afectan de manera significativa las finanzas del desarrollo inmobiliario). Nuevamente, el modelo de gestión no prevé conflictos o negociaciones sociales, incluso tratándose de habitantes de las zonas residenciales exclusivas, con lo que podemos retomar la discusión inicial de Santos en el sentido de que las verticalidades globales predominan sobre las horizontalidades locales, reafirmando con ello la proposición inicial de Santa Fe como dispositivo activo de la globalización.

Un elemento que está desempeñando una función clave en los dispositivos de la globalización es la seguridad. Evidentemente los circuitos de capital requieren la garantía de que sus intereses están a buen resguardo, por lo que en el caso de Santa $\mathrm{Fe}$, la Asociación diseñó un "Operativo Santa Fe", que comprende la coordinación de sesenta efectivos de la policía bancaria y comercial a cargo de diez patrullas y con 
un sistema integrado de cámaras de circuito cerrado de televisión dependiente de la Secretaría de Seguridad Pública del Distrito Federal. Al interior de los complejos residenciales, los expertos en sistemas de seguridad sugieren "reglas básicas de seguridad condominal" en las que no solo recomiendan denunciar actos de violencia, sino que insisten de manera enfática en la identificación de sujetos de apariencia "sospechosa o subversiva" que pudieran poner en peligro la tranquilidad del vecindario (Boletin Colonos Santa Fe, 2011, p. 14). Entre otras cosas, el consultor recomienda - naturalmente - contratar una empresa de seguridad privada que sea "capaz de enfrentar cualquier contingencia que ponga en riesgo la integridad de los habitantes de esa unidad" (es inimaginable, entonces, la intervención de la policía regular para estas situaciones). Sin embargo, lo más dramático quizá no sea la esquizofrenia hacia el exterior, sino las recomendaciones para el interior de los condominios: se sugiere construir un muro que impida la visibilidad, tener perros de ataque, videovigilancia, puertas sólidas, ventanas enrejadas e, incluso, la construcción de un refugio interior en el área de dormitorio asegurado con una puerta de acero, desde donde activar el botón de pánico (Boletín Colonos Santa Fe, 2011, p. 14).

\section{La recuperación ecológica como soporte de inversión inmobiliaria}

El proyecto urbano Santa Fe se inició con la donación de 20 hectáreas por parte del gobierno federal para la construcción de la Universidad Iberoamericana, y 23 hectáreas para el Centro Comercial Santa Fe (en su momento, el mayor de América Latina), generando un cambio en las densidades y usos de la zona. Lo anterior se tradujo en un aumento explosivo del precio del suelo en corto tiempo, integrando lo que Samuel Jaramillo (2003) denomina el valor agregado de los usos potenciales del predio (p. 20). De acuerdo con Gamboa de Buen (1994) - como promotor inicial del proyecto-, los objetivos centrales de la creación de Santa Fe fueron tres: la recuperación ecológica de la zona, la creación de empleos y la transformación de la zona en un centro de inversiones inmobiliarias. A finales del sexenio salinista, consideraba además que la zona se había convertido en "un verdadero centro urbano con todos los usos (oficinas, comercio, vivienda, entretenimiento, salud y educación), convirtiéndose en un icono para la ciudad de México" (Gamboa de Buen, 2006). Es interesante la referencia a iconos tratándose de operaciones urbanas de gran escala. Por definición, los iconos son un signo que sustituye al objeto mediante su representación, y frecuentemente se convierten en objetos de culto, o bien en sitios con un simbolismo hegemónico.

En retrospectiva, el promotor del desarrollo reflexiona en que de no haberse creado Santa Fe, "hubiera sido ocupada poco a poco y de manera desordenada para ir resolviendo situaciones de corto plazo. [Por ello] ante semejante perspectiva, se optó por un proyecto de desarrollo claro, ordenado y autofinanciable" (Gamboa de Buen, 1994). Sin embargo, es más ilustrativo el hecho de que en su análisis reconozca una falta de integración social con el resto de la ciudad, una administración urbana inoperante, así como una conectividad deficiente respecto de la mancha urbana y a los sistemas de transporte público metropolitano. 
Si bien autores como Pérez Negrete (2009, p. 37) han argumentado que los planificadores originales de Santa Fe no tenían intención de amurallar la ciudad o generar una mayor segregación socioespacial, lo cierto es que el precio del suelo, las dimensiones mínimas de los lotes, la calidad constructiva y el uso del suelo propiciaron la concentración de desarrollos inmobiliarios y corporativos de lujo, que al lado de los barrios populares de la zona hicieron evidente la extrema polarización del ingreso. Al respecto comenta Jordi Borja en entrevista con Valenzuela (2006b): "Este tipo de barrios cerrados son una doble expresión: por una parte, de la negación de la ciudad como lugar de intercambio de personas iguales (o por lo menos, formalmente iguales); de la ciudad, por tanto, como lugar de vida colectiva, pero también es la expresión de una sociedad desigual, en que los grupos privilegiados manifiestan esta injusticia, ya que la expresan precisamente con su miedo, porque se saben cómplices de una sociedad terriblemente injusta” (p. 74).

Entre los objetivos enunciados en el Programa Parcial de Desarrollo Urbano de Santa Fe destaca la creación de un polo de articulación financiera y comercial, tanto a escala nacional como internacional. No obstante, el programa también contemplaba un centro urbano donde la mezcla de usos mixtos, actividades económicas y actividades sociales contribuyera en la construcción de la identidad del lugar. Además de dicho centro, se planteó la inclusión de dos parques públicos - la Alameda Poniente y Prados de la Montaña-, los que fueron pensados para el esparcimiento y convivencia de los habitantes. Estos espacios nunca pudieron ser utilizados debido a que se construyeron sobre rellenos sanitarios y no fueron objeto de una descontaminación del suelo. Como consecuencia, además de seguir expidiéndose gases lixiviados (tóxicos), el calentamiento del suelo impidió el crecimiento de vegetación alguna.

Del mismo modo en que Santa Fe fue presentado como un proyecto estratégico de recuperación ambiental, otras operaciones urbanas similares se han llevado a cabo en zonas desfavorecidas de otras ciudades (basureros, cañadas, ex zonas industriales, minas de arena), pero que tienen una posición estratégica, y es esta accesibilidad la que les otorga su potencial de desarrollo y para el acomodo de nuevos usos. Si bien Santa Fe se ubicó en suelos arenosos y en el sitio de un antiguo basurero de la ciudad, también es cierto que su colindancia con las zonas residenciales de lujo al poniente de la ciudad fue determinante para su creación.

\section{Los circuitos globales del capital inmobiliario}

Aunque estudios recientes ubican a Ciudad de México entre las primeras veinte ciudades globales y la que encabeza la lista en América Latina (Parnreiter, 2002, p. 19), lo cierto es que lo global de la ciudad está altamente concentrado y fragmentado en el tejido urbano. En el caso de Santa Fe, tanto las nuevas geografías de centralidad como la construcción de unidades territoriales responden a configuraciones de carácter económico y financiero, lo que Sassen (2003) califica como un "reescalamiento de los territorios estratégicos que articulan el nuevo sistema” (p. 1). La di- 
ferenciación territorial es central dentro del esquema económico de Ciudad de México, de manera que los flujos hegemónicos se localizan sobre Paseo de la Reforma, Avenida Juárez, Santa Fe, Polanco, Insurgentes Sur y Periférico Sur, que son zonas o corredores financiero-comerciales de alto valor inmobiliario (Parnreiter, 2002, p. 24). Ocurre, así, que las ciudades no adquieren un carácter global integral, sino que concentran funciones que se articulan dentro del entramado económico, financiero y comercial de los circuitos del capital, pero que guardan poca relación con el tejido urbano local. De acuerdo con Castells y Hall (1994), la economía global se estructura por medio de centros direccionales al interior de redes de intercambio nacional e internacionales, que permiten articular, gestionar y potenciar las actividades de las empresas (p. 157).

Si bien autores como Messmacher (1987, p. 24) o Kresl y Gappert (1995, p. 318) están convencidos de que la era global y de la información tendrá un lugar predominante en las megaciudades, estas presentan una discontinuidad territorial segregada y paradójica. Como plantean Borja y Castells (1997), "las megaciudades [son] constelaciones territoriales discontinuas hechas de fragmentos espaciales, de parcelas funcionales y de segmentos sociales" (pp. 49-50), del mismo modo que los grandes proyectos urbanos como Santa Fe en México, Nordelta o Puerto Madero en Buenos Aires, están constituidos por fragmentos territoriales autocontenidos, donde la funcionalidad se reduce a la gestión de infraestructuras y servicios (sin pretensiones de representación y democracia) y la composición social se manifiesta mediante una diferenciación inequívoca entre los dueños del capital y sus asalariados.

Tomando en consideración lo anterior, puede afirmarse que Santa Fe materializa los requisitos indispensables para articularse como dispositivo de la economía global, el cual comprende "la constitución de un nodo urbano de gestión de servicios avanzados, organizados invariablemente en torno a un aeropuerto internacional; un sistema de telecomunicaciones por satélite; hoteles de lujo, con seguridad adecuada; servicios de asistencia secretarial en inglés; empresas financieras y de consultoría con conocimiento de la región; oficinas de los gobiernos regionales y locales capaces de proporcionar información e infraestructura de apoyo a la inversión internacional; y un mercado de trabajo local con personal calificado en servicios avanzados e infraestructura tecnológica" (Borja \& Castells, 1997, p. 37). El inicio de Santa Fe contemplaba en su segunda fase la construcción de 2.500 viviendas sobre una superficie de casi un millón de metros cuadrados, con tres modalidades residenciales: lotes unifamiliares de 350 metros cuadrados, clusters para condominios horizontales y lotes para torres de condominios. Sin embargo, en el origen del proyecto siempre existieron cuestionamientos puntuales respecto del impacto que los proyectos urbanos metropolitanos pudieran tener para el mejoramiento de la calidad de vida de la población. Al respecto, un participante en las mesas de discusión de la segunda etapa del proyecto apuntaba a sus limitantes, destacando que podríamos estar simplemente impulsando "el desarrollo del subdesarrollo y que el impacto consista nada más en la expulsión de gente pobre y que se queden algunas personas; por ejemplo, algunas muchachas, en lugar de trabajar de sirvientas, en 
lugar de estar vendiendo en la banqueta algunas baratijas, puedan traer un delantal bien almidonado atendiendo en el restaurante VIPs y que eso se considere que es desarrollo y que así se está contribuyendo a la solución de los grandes problemas nacionales" (Ramírez \& Stolarski, 1993, p. 168).

México - como la mayoría de los países en América Latina en la década de los ochenta- sufrió los efectos de los ajustes estructurales de la economía impuestos por el Fondo Monetario Internacional, los cuales derivaron en la desregulación, las privatizaciones y el libre mercado. Inmersos en un mundo globalizado, el sector inmobiliario es uno de los que más resienten las fluctuaciones económicas mundiales (Bardhan, Edelstein \& Tsang, 2008). Fue así que la reciente crisis financiera norteamericana de mediados de 2008 se hizo sentir poco después en Santa Fe, donde las inversiones en el sector inmobiliario cayeron en 94\%, según estimaciones del corporativo ARX Patrimonium (La Jornada, 2009). El desplome fue vertiginoso, ya que una inversión de 9.455 millones de dólares en 2008 se redujo a 597 millones en los primeros diez meses de 2009, lo cual provocó el cierre o interrupción de numerosas obras y proyectos.

Santa Fe se afirma, entonces, como una respuesta territorial al Tratado de Libre Comercio firmado en 1994 con los Estados Unidos y Canadá y que requería los vectores de integración jerárquica regulada que propone Milton Santos ${ }^{2}$. Dichos sectores socioeconómicos globalizados crecen a partir de su integración a la red de lugares estratégicos y son, por tanto, un resultado directo de la organización de la economía global (Sassen, 2003, p. 15). La geografía de la globalización se articula, entonces, mediante espacios definidos y altamente estructurados, profundizando con ello la discontinuidad espacial. No obstante, esta segregación respecto del conjunto del tejido urbano de la ciudad, que incluye sofisticados sistemas de comunicaciones - como carreteras, trenes rápidos y aeropuertos-, frecuentemente viene a ser banalizada como parte del costo del progreso y procesos civilizatorios de la globalización.

Las ciudades en la globalización no han servido, entonces, para redefinir jerarquías o superar desigualdades espaciales, sino que incluso tienden a desconectarse territorialmente de la región circundante. Esto resulta paradójico dentro de los planteamientos clásicos del desarrollo regional, según el cual los sistemas urbanos servirían precisamente para instrumentar la integración territorial de las economías regionales (Sassen, 2003, pp. 15-16). Por tanto, la economía global se mueve, hasta cierto punto, dentro de circuitos independientes de las problemáticas socioeconómicas del territorio en que se desenvuelven. Los sectores involucrados no propician una mayor integración socioespacial sencillamente porque responden a una lógica de acumulación de capital poderosa y bien articulada con los otros nodos globales. De acuerdo con Sassen (2003), para que las ciudades al interior de los circuitos globales desarrollen las funciones correspondientes, es necesario que los órdenes económico, político y espacial de dichas ciudades se transformen, aun cuando di-

2 Si bien Santa Fe se creó en los años ochenta, un impulso detonador fue la modificación del artículo 27 constitucional, que, de acuerdo con Parnreiter (2011, p. 17), permitió el boom inmobiliario de los años noventa. 
námicas tradicionales permanezcan vigentes en paralelo (p. 26). En el caso de América Latina, y de México en particular, no fue necesario un "nuevo orden", sino que simplemente se profundizaron las tendencias históricas de polarización del ingreso, se legitimaron las operaciones urbanísticas en nombre de la competitividad y se consolidaron los territorios de exclusividad como únicos entornos posibles para el capital en una sociedad "partida" y desigual (Ventura, 2000).

\section{A manera de conclusión}

La distinción entre los flujos verticales y horizontales que propone Santos (1993, p. 74) refleja de manera cabal la estructura socioespacial generada por dispositivos de la globalización, como es el caso de Santa Fe en México. Mientras que el tejido local horizontal recibe los flujos hegemonizados del capital, los puntos de contacto en sentido vertical reciben los flujos hegemónicos de los circuitos globales del capital global. Si bien la morfología urbana resultante es comparable con otros puntos de contacto localizados en Zurich, São Paulo o Buenos Aires, los dispositivos no generan coherencia espacial, sostenibilidad ambiental o articulación territorial (Castells \& Borja, 1997, p. 61).

Siguiendo el análisis de Gramsci (1971, p. 58) sobre la hegemonía como un mecanismo de dominio en el cual se busca un liderazgo moral e ideológico, tenemos que megaproyectos corporativos como Santa Fe surgieron en el periodo que Harvey $(1989$, p. 4) señala como aquel donde la gestión urbana pasó de ser gerencial a empresarial (finales de los ochenta), convirtiéndose de paso en modelos a los cuales seguir. En México, a pesar de las crisis económicas recurrentes por las que atravesó el país, el espejismo globalizador dio lugar a dispositivos como estos, que vendrían a fortalecer a las elites políticas y económicas, al mismo tiempo que el enfoque economicista neoliberal daba el sustento teórico a la priorización del capital por encima del bienestar social.

Uno de los problemas centrales en Santa Fe ha sido la identidad social. Si las identidades se construyen por la representación y por medio de distintas y variadas relaciones con las instituciones (tanto del Estado como de la sociedad civil), ocurre que en el caso de Santa Fe existen limitados mecanismos para que la población en su conjunto se vea representada en un proyecto de esta naturaleza y pueda establecer una identidad incluso en contra de una sociedad jerárquicamente organizada. Quizá los valores hegemónicos empresariales - en el sentido gramsciano- hayan permeado el sector laboral y la gente común se sienta inclinada hasta cierto punto a tolerar e incluso apoyar un proyecto globalizante del cual obtiene magros beneficios.

En una lógica neoliberal, la puesta en operación de megaproyectos con la participación de grandes capitales e inversiones externas, sirve como herramienta de progreso para potenciar la competitividad internacional y alcanzar un posicionamiento global. Sin embargo, la construcción de equipamientos urbanos, desarrollos residenciales e infraestructuras mayores para recibir dichas operaciones, profundiza las divisiones socioterritoriales en la ciudad, evitando incluso su articulación con el tejido urbano 
circundante o con mecanismos de gestión y democracia que permitan la construcción de un tejido social sano. En ese sentido, las formas privatizadas de gestión, autónomas y elitistas, no surgen como una respuesta a la ineficiencia de la administración pública, sino que expresan la renuncia a la responsabilidad del gobierno para dirigir el desarrollo de la ciudad de manera equilibrada, democrática y justa.

Aun cuando Ciudad de México está considerada dentro de los nuevos centros regionales globales (Parnreiter, 2002; Castells \& Borja, 1997; Sassen 1991, 2003), esto no obsta para que grandes fragmentos del territorio se mantengan en la pobreza extrema y con grandes carencias de infraestructuras y servicios básicos. La construcción de Santa Fe como enclave urbano de alto nivel se fue estructurando paulatinamente para proveer no solo una sede para las corporaciones multinacionales, sino el mejor de los mundos para las elites económicas y sociales: centros privados de educación media y universitaria, complejos residenciales con todos los servicios, centros comerciales de primer nivel, así como la cercanía a los centros de poder y los círculos sociales más exclusivos de Ciudad de México. Por tanto, Santa Fe responde a una lógica de los circuitos de capital no solo financiero, sino también inmobiliario. Los flujos hegemónicos se fortalecen con estos "dispositivos globales", al tiempo que se legitiman y establecen como parte de un proyecto hegemónico de alcance global. @EURE

\section{Referencias bibliográficas}

Akele T. (2007). Presenta Gicsa proyecto de Ciudad Santa Fe. Extraído el 12 de enero de 2011 de http:// www.esmas.metroscubicos.com.

Bardhan, A., Edelstein, R. \& Tsang, D. (2008). Global economic and financial integration and real estate security returns. Real Estate Economics, 36(2), 285-311.

Barros Nock, M. (1993). Programa Santa Fe. En A. Ramírez \& N. Stolarski, Proyectos urbanos metropolitanos. Los retos de la Ciudad de México (pp. 123-130). México: Editorial Cambio XXI.

Boletin Colonos Santa Fe, 2(7) (febrero-marzo, 2011). Extraído el 20 de octubre de 2011, de http:// www.colonossantafe.com/wp-content/uploads/2011/05/2011-febrero-marzo.pdf

Castells, M. \& Borja, J. (1997). Local y global. La gestión de las ciudades en la era de la información. Madrid: Taurus.

Castells, M. \& Hall, P. (1994). Technopoles of the World: The making of twenty-first-century industrial complexes. Londres: Routledge.

Castells, M. (1979). La intervención administrativa en los centros urbanos de las grandes ciudades. Papers, Revista de Sociología, 11, 227-250.

Colonos Santa Fe. Extraído el 4 de febrero de 2011, de http://www.colonossantafe.com/historial.html

Cornejo, I. \& Bellon, E. (2001) Prácticas culturales de apropiación simbólica en el Centro Comercial Santa Fe. Convergencia, 8(24), 67-86.

Gamboa de Buen, J. (2006). The Santa Fe node: Its beginnings. En Urban Age Mexico City Conference. Extraído el 14 de enero de 2011, de http://www.urban-age.net/0_downloads/pdf.../ Mexico/D-1deBuen.pdf

Gamboa de Buen, J. (2004). Ciudad de México. Una visión. México: Fondo de Cultura Económica. 
García Fonseca, C. (2003). Las crisis recurrentes del peso mexicano: Causas, efectos y posibles soluciones. Tesis doctoral. Universidad Veracruzana/Universidad de Barcelona.

Gramsci, A. (1984). Los intelectuales y la organización de la cultura. Trad. Raúl Sciarreta. Buenos Aires: Nueva Visión.

Gramsci, A. (1971). Selections from the Prison Notebooks. Londres: Lawrence \& Wishart.

Hall, P. (1998). Cities in Civilization. Londres: Weidenfeldand Nicolson.

Hall, P. (1995). Towards a general urban theory. En J. F. Brotchie, M. Batty, E. Blakely, P. Hall, P. W.

Newton (Eds.), Cities in Competition. Productive and sustainable cities for the $21^{\text {st }}$ century

(pp. 3-32). Melbourne: Longman Australia.

Harvey, D. (2007). Neoliberalism as creative destruction. The Annals of the American Academy of Political and Social Science, 610(1), 21-44. doi:10.1177/0002716206296780

Harvey, D. (1989). From managerialism to entrepreneurialism: The transformation in urban governance in late capitalism. Geografiska Annaler. Series B, Human Geography, 71(1), The Roots of Geographical Change: 1973 to the Present, 3-17. http://www.jstor.org/stable/490503

Hoetmer, R. (2009). Democracia y movimientos sociales en un mundo de globalizaciones: Hacia concepciones contrahegemónicas. En E. Tejada Sánchez (Ed.), Movimientos sociales y democracia en el Perú de hoy. Reflexiones a propósito de la gesta de Arequipa. Arequipa: Centro de Estudiantes de Sociología de la Universidad Nacional de San Agustín (UNSA), Facultad de Ciencias Histórico Sociales de la UNSA y Programa Democracia y Transformación Global.

Jaramillo, S. (2003). Los fundamentos económicos de la participación de plusvalias. Bogotá/Cambridge: Ed. Universidad de los Andes y Lincoln Institute of Land Policy.

Jones, G. \& Moreno-Carranco, M. (2007). Megaprojects: Beneath the pavement, excess. City, 11(2), 144-164, doi: 10.1080/13604810701395969

Kresl K. \& Gappert, G. (Eds.). (1995). North American Cities and the Global Economy. Thousand Oaks, CA: Sage.

La Jornada. Raúl Llanos Samaniego (2010a). Vecinos de zonas populares amenazan con derribar estructuras. 30 de agosto.

La Jornada. Raúl Llanos Samaniego (2010b). Desaparecerá el Fideicomiso Colonos Santa Fe: Luna. 27 de diciembre.

La Jornada. Laura Gómez Flores (2009). Cae en un año 94\% la inversión del sector inmobiliario: Expertos. 9 de noviembre.

La Jornada. José González Martínez (2005). En Cámara Lenta. 19 de septiembre.

López Zárate M. \& Ochoa Méndez, J. (1995). Santa Fe, razones de un proyecto. Ciudades, 27 (julioseptiembre) (Red de Investigación Urbana [RNIU], México).

Luna, M., Millán, R. \& Tirado, R. (1985). Los empresarios en el inicio del gobierno de Miguel de la Madrid. Revista Mexicana de Sociología, 47(4), 215-257.

Lungo, M. (2004). Grandes proyectos urbanos. Una visión general. En M. Lungo (Ed.), Grandes proyectos urbanos (pp. 15-67). El Salvador: UCA Editores/Lincoln Institute of Land Policy.

Mattos, C. A. de (2001). Movimientos del capital y expansión metropolitana en las economías emergentes Latinoamericanas. Revista de Estudios Regionales, 60, 15-43.

Merriam-Webster Dictionary (1993). 10th ed. Springfield, MA: Merriam-Webster, Inc.

Messmacher, M. (1987). México, megalópolis: Evolución y dinámica de los municipios conurbados de la Ciudad de México. México: Secretaría de Educación Pública.

Parnreiter, Ch. (2011). Formación de la ciudad global, economía inmobiliaria y transnacionalización 
de espacios urbanos. El caso de Ciudad de México. EURE (Santiago), 37(111), 5-24. doi: 10.4067/S0250-71612011000200001

Parnreiter, Ch. (2002). Ciudad de México: el camino hacia una ciudad global. EURE (Santiago), 28(85), 89-119. doi: 10.4067/S0250-71612002008500006

Peninsula Santa Fe. Extraído el 18 de abril de 2011, de http://peninsulasantafe.mx.

Pérez Negrete, M. (2009). Santa Fe: ¿Cómo se materializa la utopía de la transformación urbana? Iberoforum, Revista de Ciencias Sociales de la Universidad Iberoamericana, 4(7)(enero-junio).

Perló Cohen, M. (1999). Desastres en las grandes ciudades. México: Red Mexicana de Estudios Interdisciplinarios para la Protección de Desastres.

Reforma. Pilar Gutiérrez (2010). Cuajimalpa denuncia a exfuncionarios por City Santa Fe. 1 de marzo.

Ramírez H., Stolarski, G. \& Stolarski, N. (Comps.). (1993). Proyectos urbanos metropolitanos. México: Fundación Distrito Federal, Cambio XXI.

Salinas Callejas, E. \& Tavera Cortés, M. E. (2007). La transición de la economía mexicana 1982-2004. En G. Vidal \& A. Guillén R. (Comps.), Repensar la teoría del desarrollo en un contexto de globalización. Homenaje a Celso Furtado (pp. 275-290). Buenos Aires: Consejo Latinoamericano de Ciencias Sociales (Clacso), Universidad Autónoma Metropolitana, México; Red Eurolatinoamericana de Estudios sobre el Desarrollo Celso Furtado.

Santos, M. (1993). Los espacios de la globalización. Anales de Geografía de la Universidad Complutense, 13, 69-77.

Sassen, S. (2003). Localizando ciudades en circuitos globales. EURE (Santiago), 29(88), 5-27. doi: 10.4067/S0250-71612003008800001

Sassen, S. (1991).The Global City. New York, London, Tokyo. Princeton, NJ: Princeton University Press.

Servicios Metroplitanos (Servimet). (1990). Plan Maestro para la Zedec Santa Fe. México: Departamento del Distrito Federal.

Stahl, K. (1994). Política social en América Latina. La privatización de la crisis. Nueva Sociedad, 131 (mayo-junio), 48-71.

Valenzuela, A. (2011). Mexico City: Power, equity and sustainable development. En A. Sorensen \& J. Okata (Eds.), Megacities: Urban form, governance, and sustainability. Londres \& Tokio: Springer.

Valenzuela, A. (2007). Santa Fe (México): Megaproyectos para una ciudad dividida. Cuadernos Geográficos, $40(1), 53-66$.

Valenzuela, A. (2006a). Mexico City and the places of globalization. En SUR, Center for Sustainable Urban Regeneration, University of Tokyo, Japan.

Valenzuela, A. (2006b). Ciudades, política y poder. Entrevistas a Jordi Borja, François Choay y Noam Chomsky, Replicante, 11(7) (mayo-julio), 72-77.

Ventura, Z. (1994). Cidade partida. São Paulo: Companhia das Letras.

Villalpando, R. (1993). El proyecto Alameda en el contexto del desarrollo urbano de la Ciudad de México. En G. Ramírez H. \& N. Stolarski R. (Comps.), Proyectos urbanos metropolitanos. México: Fundación Distrito Federal, Cambio XXI. 